\title{
PARAMONE
}

EDITIONEN UND AUFSÄTZE

VON MITGLIEDERN DES HEIDELBERGER

INSTITUTS FÜR PAPYROLOGIE

ZWISCHEN 1982 UND 2004 


\title{
ARCHIV FÜR PAPYRUSFORSCHUNG UND VERWANDTE GEBIETE
}

\author{
BEGRÜNDET VON ULRICH WILCKEN
}

\section{BEIHEFT 16}

\section{PARAMONE}

EDITIONEN UND AUFSÄTZE

VON MITGLIEDERN DES HEIDELBERGER

INSTITUTS FÜR PAPYROLOGIE

ZWISCHEN 1982 UND 2004

\author{
HERAUSGEGEBEN VON \\ BÄRBEL KRAMER WOLFGANG LUPPE \\ HERWIG MAEHLER GÜNTER POETHKE
}

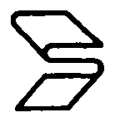

K · G · SAUR MÜNCHEN · LEIPZIG 


\title{
PARAMONE
}

\section{EDITIONEN UND AUFSÄTZE VON MITGLIEDERN DES HEIDELBERGER INSTITUTS FÜR PAPYROLOGIE ZWISCHEN 1982 UND 2004}

\author{
HERAUSGEGEBEN \\ VON \\ JAMES M. S. COWEY UND \\ BÄRBEL KRAMER
}

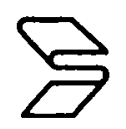

K · G · SAUR MÜNCHEN · LEIPZIG 2004 


\title{
ARCHIV FÜR PAPYRUSFORSCHUNG VERLAG K · G · SAUR MÜNCHEN · LEIPZIG
}

\author{
In Verbindung mit den Staatlichen Museen zu Berlin \\ - Preußischer Kulturbesitz -
}

Das „Archiv für Papyrusforschung und verwandte Gebiete“ (APF) wurde 1901 von Ulrich Wilcken im Verlag B. G. Teubner begründet.

Das APF erscheint in zwei Halbjahresheften im Gesamtumfang von durchschnittlich 28 Bogen. Bezugspreis $€$ 188,- inklusive Versandkosten.

Bestellungen durch alle Buchhandlungen wie auch beim Verlag:

K. G. Saur Verlag, Postfach 701620 - D-81316 München · http://www.saur.de e-mail: saur.info@thomson.com

Herausgeber:

Prof. Dr. B. Kramer, Universität Trier, Fachbereich III, Papyrologie, 54286 Trier Prof. Dr. W. Luppe, Martin-Luther-Universität, Institut für Klassische Altertumswissenschaften, Universitätsplatz 12, 06099 Halle

Prof. Dr. H. Maehler, University College London, Department of Greek and Latin, Gower Street, London WCIE 6BT

Prof. Dr. G. Poethke, Ägyptisches Museum und Papyrussammlung

Staatliche Museen zu Berlin - Preußischer Kulturbesitz Schloßstr. 70, 14059 Berlin

Bibliografische Information Der Deutschen Bibliothek

Die Deutsche Bibliothek verzeichnet diese Publikation in der Deutschen Nationalbibliografie; detaillierte bibliografische Daten sind im Internet über http:/dnb.ddb.de abrufbar.

(C) 2004 by K. G. Saur Verlag GmbH, München und Leipzig Printed in Germany

Alle Rechte vorbehalten. All Rights Strictly Reserved.

Jede Art der Vervielfältigung ohne Erlaubnis des Verlages ist unzulässig.

Gesamtherstellung: Druckhaus „Thomas Müntzer" GmbH, 99947 Bad Langensalza ISBN 3-598-77591-1 